\title{
A Tool for Replay and Analysis of Gaze-Enhanced Multiparty Sessions Captured in Immersive Collaborative Environments
}

\author{
Alessio Murgia \\ School of Systems \\ Engineering \\ University of Reading \\ Paul Sharkey \\ School of Systems \\ Engineering \\ University of Reading
}

Anthony Steed

Department of Computer

Science

University College London

\{a.murgia,p.m.sharkey\}@reading.ac.uk; \{r.wolff,d.j.roberts\}@salford.ac.uk; \{w.steptoe, a.steed\}@cs.ucl.ac.uk; \{e.guimaraes,j.rae\}@roehampton.ac.uk

\author{
Robin Wolff \\ The Centre for Virtual \\ Environments \\ University of Salford \\ David Roberts \\ The Centre for Virtual \\ Environments \\ University of Salford
}

\author{
William Steptoe \\ Department of Computer \\ Science \\ University College London
}

Estefania Guimaraes

School of Human and Life

Sciences

Roehampton University

\begin{abstract}
A desktop tool for replay and analysis of gazeenhanced multiparty virtual collaborative sessions is described. We linked three CAVE ${ }^{T M}$-like environments, creating a multiparty collaborative virtual space where avatars are animated with $3 D$ gaze as well as head and hand motions in real time. Log files are recorded for subsequent playback and analysis using the proposed software tool.

During replaying the user can rotate the viewpoint and navigate in the simulated $3 D$ scene. The playback mechanism relies on multiple distributed log files captured at every site. This structure enables an observer to experience latencies of movement and information transfer for every site as this is important for conversation analysis. Playback uses an eventreplay algorithm, modified to allow fast traversal of the scene by selective rendering of nodes, and to simulate fast random access. The tool's analysis module can show each participant's $3 D$ gaze points and areas where gaze has been concentrated.
\end{abstract}

\section{Introduction}

Collaborative virtual environments (CVEs) allow co-located or remote participants to experience communication inside a rich social, spatial and informational context $[1,2]$. Co-presence amongst remote participants is achieved by enabling local sites to access a shared virtual environment. In these environments users are embodied, or represented, as avatars. It is thus possible for each participant to experience a sense of being in another place while interacting with other individuals and with the surrounding objects.

We have developed the first CVE system (referred to as EyeCVE) that provides eye-tracking capability to each participant and uses it to drive avatar gaze in realtime in a $\mathrm{CAVE}^{\mathrm{TM}}$-like environment. Remote participants can see and interact with their counterparts across the remote sites in the form of avatars, while changes to the virtual environment are updated in realtime. Participant's head and hand movements are also used to animate each participant's avatar [3].

In our research we are interested in analyzing how gaze affects communication in immersive CVEs [4]. One of the methods used is 'conversation analysis' [5] which exposes systematic practices, verbal and non-verbal, during human interaction and analyzes how actions are routinely carried out in this context. The analysis extracts information related, for example, to the use of gaze, turn-talking and participation. This analysis requires replaying the events that took part during the session. To facilitate this process a logging system was put in place to record events occurring in the environment in order to play them back during a later analytical stage. We present in this paper a desktop tool for analyzing and playing back multiparty collaborative sessions across virtual environments that is cross- 
platform, random-access, lightweight and open-source and thus can potentially be adopted by other CVE users.

The main contributions of this work are the development of a desktop tool that can analyze 3D gaze while playing back the recorded session as a 3D simulation, the tool's ability to provide a $3^{\text {rd }}$ party viewpoint and to allow navigation in the environment while replaying the scene, and the open-source and portable nature of this tool that make it portable from desktop to a CAVE environment. This last feature would allow users to immerse themselves in the session as if they were participating in it as invisible observers.

The paper structure consists of a review of related work (section 2), a brief description of our CVE system and relative logging routine (section 3 ), a description of the analysis methods implemented to date together with the software structure that embeds them and plays back the $\log$ files (section 4).

\section{Related Work}

Recording collaborative sessions is increasingly seen as a necessity in computer supported cooperative work (CSCW) [6, 7]. Generic algorithms for efficient recording and playback of audio, video and data streams have become a development priority due to the large number of data generated in these applications [8].

Architectures capable of recording CVEs sessions have been formulated based on sequential or randomaccess, and as well as being based on proprietary software they are generally bound to the system on which they have been developed. Typical examples are the architectures implemented for the MASSIVE-3 system [9] and the DIVE system [10, 11]. Systemindependent architectures as that presented by Hosseini and Georganas based on the MPEG-4 format [12] allow sharing recorded data across multiple clients based on a common format. In this specific application only sequential access was implemented.

From a scene management point of view, a virtual environment consists of infrequent, but large changes to the scene graph such as the introduction of new objects, combined with frequent but small changes such as the movement of objects. One common approach is to serialize the full scene state infrequently and then record all change events as they happen $[6,10,13]$. In these applications random access is achieved by loading the full scene immediately preceding the requested moment in time and updating the scene based on the partial recordings (if any exist) taken between the full recording and the requested moment. This approach tends to generate large repository files.

In our approach we decided to minimize the repository files using an event-replay mechanism where each event-change is recorded sequentially together with a timestamp [6, 14, 15]. Random access is implemented by playing the events sequentially up to the requested point. The drawback of this mechanism is that delays can be generated before the requested position is reached. However in our approach we minimized these delays by proposing a selective updating of the scene (see section 4.4). In addition, our application was designed not only to playback sessions but also as a tool to analyze $3 \mathrm{D}$ gaze information recorded on $\log$ file.

Gaze analysis has been applied to different fields, for example to evaluate where avatars animated using pre-recorded gaze are looking at in an immersive environment [16], to assess the effectiveness of eye-vsmouse interfaces [17], to measure the impact of new visualization technologies [18], to train the inspection of mechanical structures performed in virtual reality [19], to evaluate skill learning [20,21], to study human communication [22], visual attention [23], to improve teleconferencing [24], and to evaluate the usability of virtual environments through the study of breaks in presence [11]. In our research we are particularly interested in understanding how gaze-enhanced virtual sessions support communication among participants compared to other more established systems such as teleconferencing [4]. For these reasons we carry out conversation and gaze analysis to extract information related to turn-talking, attention, and involvement response. In this paper we present an example of a gaze-point map that shows the 3D points of gaze, or points of regard (PoR), for each subject taking part in the virtual session. Two-dimensional gaze maps are available in commercial software. The most popular commercial tools examine the temporal distribution of 2D gaze coordinates by extracting plots such as gaze history, fixation, saccade occurrence, areas of interest and related hotspots maps, which indicate the areas mainly looked at. Typical examples are applications distributed by LC Technologies, Inc., Tobii Technology and SensoMotoric Instruments.

\section{Logging Multiparty Collaborative Sessions}

The custom-built EyeCVE software platform, used to record the multiparty collaborative sessions, integrates tracked head, hand and eye-gaze with linked immersive VR displays, such as CAVE, over the Internet. The system consists of a client software at each site, and a central "world-server". Figure 1 illustrates this process.

When logging-in the world-server provides a copy of the shared virtual world across participants and manages data communications between the sites. To increase local responsiveness we followed a distributed 
database approach, where each client holds a local copy of the world scene that is updated with local interactions and changes. These changes are then transmitted via the world-server to other sites. Thus, the main functions of the client software are to update the local copy of the world on the basis of the data communicated by the world-server, to read and communicate to the world-server the tracking data taken on the local site, and to render the scene.

Recording can be carried out either globally on the world-server, or locally at each site. The result of global recording would be a single log file of all the traffic seen by the server during the session. The second 'local' option would result in a separate log file for each site, which contained the session changes and interactions as seen by that local site (Figure 1).

For our logging we chose local log files because they capture the local participant's perspective. This perspective is useful for understanding the interactions as experienced by participants and suits the chosen methodological approach: conversation analysis.

A local $\log$ file includes effects caused by the network characteristics of the Internet, such as loss of data packets received and varying latencies. Thus only by recording what each participant experienced during the session it is possible to understand his/her communication response clearly. A typical example of where this approach is useful occurs when data packets are lost during transmission from a site ' $A$ ' to a site 'B'. When participant at site ' $\mathrm{B}$ ' only receives part of the data, a response is triggered according to the stimulus in a way that may conflict with what participant at site 'A' expects. In turn participant at site ' $A$ ' reacts by reinforcing or clarifying the original stimulus.

Similarly it also is important to observe the latency introduced when representing eye-gaze between remote sites, as well as the synchronization of eye-gaze with hand gestures. We measured on average a lag of 150 $\mathrm{ms}$ between capturing (reading from tracking device)

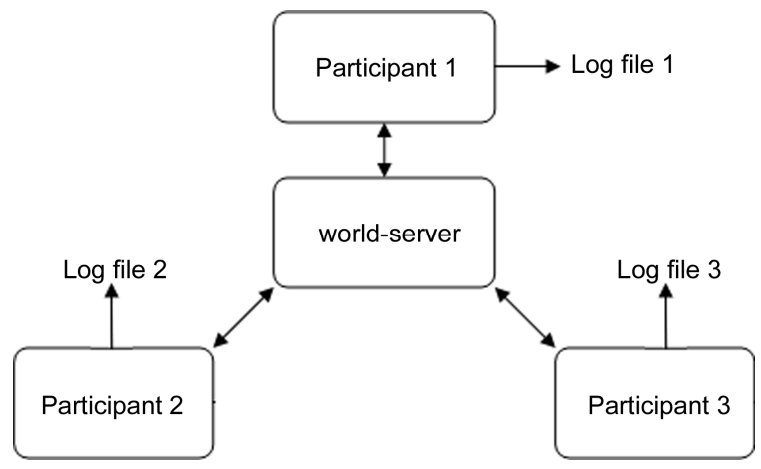

Figure 1. Client-server architecture during data recording. Components of the player application and their relative interactions. and displaying eye-tracking data on a remote site (remote display).

A local log mechanism works by recording updates when they occur on the display, that is, when they are applied to the scene graph, for example when repositioning an object or rotating an avatar's eye.

Replaying updates and changes in a virtual world from a recorded session must progress in the same time steps and order as in the original session. In our system, this is supported through a synchronized global "wallclock" time. In a typical log file each lines starts with a timestamp and is followed by a command that specifies the creation ('C') of an avatar by loading parts of a 3D model, or hierarchy changes to scene graph by attaching ('A') a node to another, or repositioning of nodes ('M'). The instruction that follows, depends on the command specified. For a node creation ('C'), these parameters are the node ID and the polygon model to be loaded. If the command is an attach instruction, children and parent node follow the attach command ('A'). Alternatively if the node is moved ('M'), 8 parameters that include $3 \mathrm{D}$ translation coordinates, quaternion axial orientation, angle of rotation about the quaternion vector and scale factor follow the move command ('M'):

5576;C;30b;robin_body_rdg.obj

5746;C;30u;robin_upper2_rdg.obj

5913;C;301;robin_lower2.obj

5914;A;30b;30

5914;A;30u;30

5915;A;301;30

5930;M;0;0.0;0.0;0.0;1.0;0.0;0.0;0.0;1.0

5930;M;1;0.0;-1.0;0.0;1.0;0.0;0.0;0.0;1.0

$5930 ; \mathrm{M} ; 27 ; 4.2 ; 0.5 ; 1.3 ; 0.0 ; 1.0 ; 0.0 ; 87.0 ; 1.0$

5930;M;27h;-0.1;3.9;0.7;-0.8;-0.4;-0.3;26.9;1.0

Figure 2 shows an example plot of a recorded session. It shows the hand movement of the avatars of two users, as well as their gaze-points.

It is easy to see that these raw-data plots do not facilitate a conversation analyst's understanding of the communication practices used during interaction between participants. This is due to lacking information on avatars' 3D location, as well as the impossibility to navigate around the scene to better observe avatars' movements and interactions.

\section{Playing Multiparty Log Files}

The analysis and replay tool (referred to as 'the player') we have developed is a desktop-based application that is open-source and cross-platform. The player interfaces with a GUI and performs rendering using the open-source library OpenSG, which was also chosen to implement the EyeCVE in the CAVE. The 


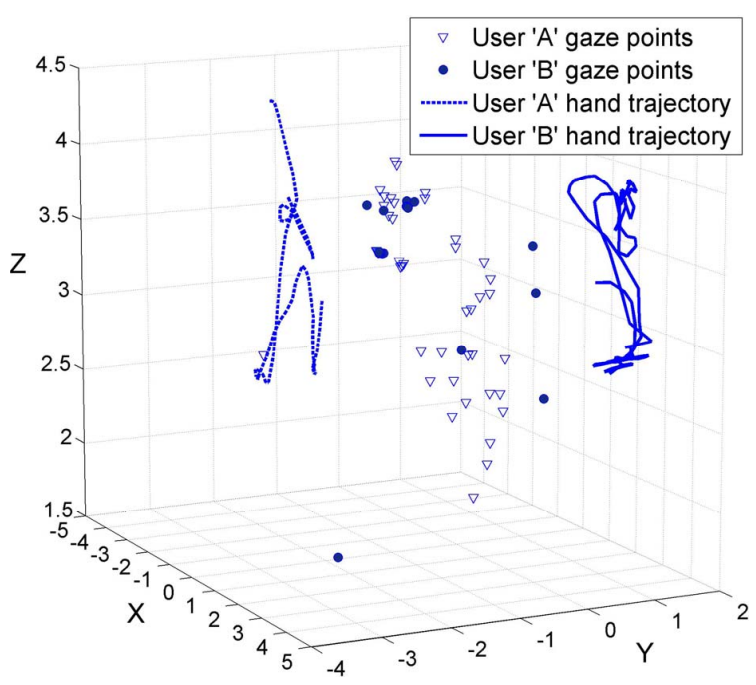

Figure 2. An extract of gaze points and hand movements plotted from log file. Data plotted in this way are not easy to read because they lack information on the locations of each avatar and on the spatial interactions between different avatars.

GUI was written using the open-source toolkit FLTK. The code was written in $\mathrm{C}++$.

The structure of the player and the different modules composing it are shown in Figure 3. The log file is read via a Tape module, while a Timer module keeps track of the real time elapsed and compares it with the time recorded on the log file. Scene updating is handled by the OpenSG Widget module. Audio of the session, if it exists, is played via an Audio module. The analysis of 3D gaze points is performed in the Analysis module. The different components are discussed in detail in the following sections.

\subsection{Reading Log Files: 'FLTK Panel' and 'Tape' Module}

The GUI interface is implemented through FLTK which handles information to and from the button groups and links to the OpenSG Widget class through which scene graph updating is done (see section 4.3).

When a $\log$ file is initially loaded, each line is parsed into a vector. When the $\log$ file is played a FLTK timeout() function invokes a read() function in the Tape class, which reads each logged line sequentially. An internal check inside the timeout() function executes the next line if the elapsed time is equal to the time difference between 2 consecutive timestamps (tdiff in Figure 4, also see log file description). This is a local check, which only supports time synchronization for a short length of time and was implemented to play the $\log$ file continuously. If this internal check were not present, and only timekeeping from the Timer class were used, the log file would play in sudden bursts, jumping to the line where the time elapsed in the $\log$ file is equal to the real time elapsed. The full synchronization procedure is described in section 4.5 .

\section{2. 'Audio' Module}

When the log file is first loaded the Audio module automatically looks for an audio .ogg file and plays it using a separate thread from that used for rendering. The open-source library OpenAL is used to handle audio playback within the application.

At this stage automatic synchronization between the 3D simulation and the audio file has not been implemented yet and the audio during the session is recorded on physical tape without using the worldserver clock, and later converted in .ogg format. A button function has been added to enable the user to play/pause the audio stream by pressing a button on the keyboard, in order to synchronize it with the 3D scene. At the moment the exact synchronization moment is found by comparing the events occurring in the scene with those taking place on an external video and audio recording taken by a camera mounted on each or the eye-tracking glasses.

\subsection{Scene Graph Updating: 'OpenSG Widget' Module}

OpenSG was chosen to implement the graphic rendering as an easily-scalable, cross-platform, scene graph-based system that sits on top of OpenGL graphic library.

OpenSG also supports a passive-window which does

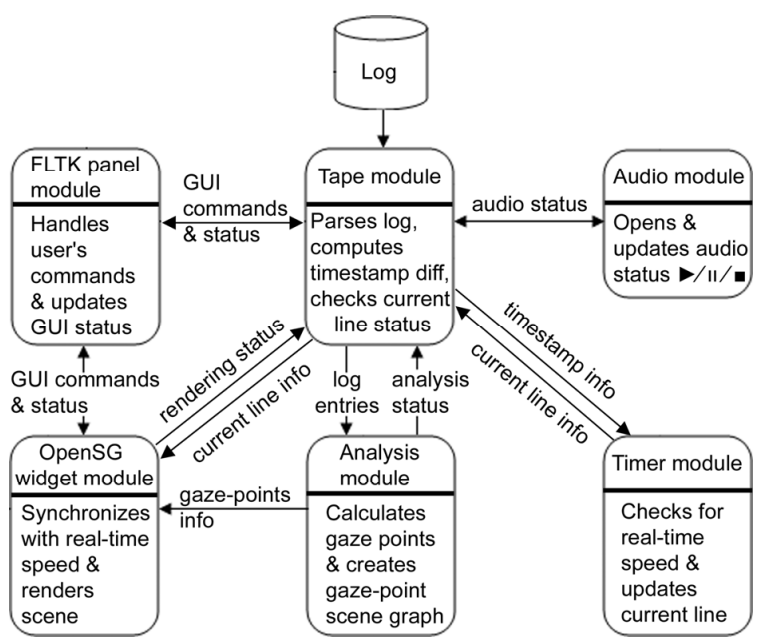

Figure 3. Components of the player application and their relative interactions. 
not handle OpenGL context and can be integrated into an existing GUI of choice (in our case FLTK) as long as the latter supports OpenGL. This means that the window where rendering occurs can be created as a derived class of the GUI window class that handles OpenGL, for FLTK this is the Fl_Gl_Window class:

class OSGWidget: public Fl_Gl_Window \{ public:

OSGWidget(int $\mathrm{X}$, int $\mathrm{Y}$, int Width, int Height, const char $*$ name $=0)$;

The OSGWidget class constitutes the core of the OpenSG widget module in Figure 3.

Mouse and keyboard commands are handled by the passive window through the definition of a handle function that replaces the virtual handle function of the parent class Fl_Gl_Window. In this way it is possible to translate, zoom and rotate the scene from a $3^{\text {rd }}$ party viewpoint, thus enabling the user to freely move around the scene and change the viewpoint.

\subsection{Scene Graph Organization and Handling}

The scene graph is created and updated dynamically (with the exception of the gaze-analysis branch, see section 4.6) every time a line from the log file is processed. After each line is read by the Tape class, the command instruction, e.g. Move node $\mathrm{X}$, is handled by the OpenSG widget module's update() function. In the scene graph structure each node is named according to its ID in the log file.

The largest delays are experienced when loading an object model the first time a node is created. The delays depend on the machine's speed and the object's complexity. For example when running the player on an Intel $^{\circledR}$ dual core 6600 at $2.4 \mathrm{GHz}$ with 2 GB RAM and 2 NVIDIA GeForce 7900 GS graphic cards, a 2256 polygons .obj file is loaded in $1375 \mathrm{~ms}$, while a 5030 polygons .obj file takes $5438 \mathrm{~ms}$.

To minimize these delays during repeated playbacks and to simulate random access more efficiently, we implemented a creation/deletion policy where a runtime check is performed every time a create command is encountered. If the log file is played forward a delete command has logical reason to exist if a node has been created beforehand. Thus if a delete command is encountered, the policy rules that the corresponding node should be made invisible to the graph traversal rather than deleted altogether from the scene graph. In this way if the node is recreated afterwards it only needs to be made visible to the traversal without reloading the associated object. In the same way, if the tape is read backwards after the node has been set to invisible, when the delete command is encountered the node is re-set to visible.
To summarize: if a delete or detach command is encountered the node is made invisible to the traversal, if reading direction is forward, or visible if playing backwards. In this way it is possible to guarantee fast traversing of the scene graph, multiple times as well as playing in reverse as the node which was once deleted needs only to be made visible again and not re-created.

Concerning move commands, the update policy is to execute them with a speed ruled by the timer module described in the following section.

\subsection{Speed Synchronization: 'Timer' Module}

Speed synchronization and random access are guaranteed via a Timer class that uses a high-resolution $(\mu \mathrm{s})$ timer. The timer is reset every time the tape is played at a different speed, or after an interruption, or if random access is invoked (see below). The total time elapsed is kept in a static variable.

The process is illustrated in Figure 4. Playing at the speed specified by the log file is done by checking the cumulative time elapsed from the moment the clock has been initialized (TE) and comparing it to the elapsed time in the log file (LTE), calculated as the sum of the time differences up to the present line.

If the ratio LTE/TE $<0.98$, i.e. if application is lagging behind by more than $2 \%$ of the total time elapsed, the Tape class invokes a member function that finds the line where $\mathrm{LTE} \geq 0.98$. This line is passed to the update() function (OpenSG widget module) which executes all the commands up to the target line within an internal 'while' cycle, ignoring the timestamp.

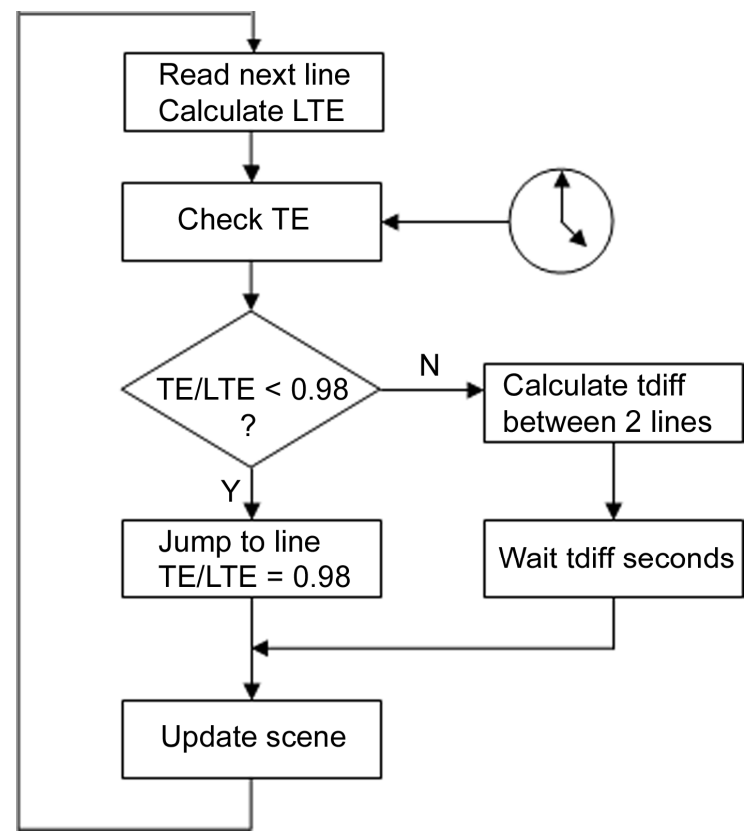

Figure 4. Real-time speed synchronization. 
To maintain the correct ratio between TE and LTE once this has exceeded 0.98, an error feedback algorithm adjusts the local time difference (tdiff in Figure 4) between 2 consecutive lines by adding to it the difference LTE - TE. This is needed to avoid the system playing at higher speed than that specified in the log file, since LTE - TE is positive if the tape is played at a quicker speed than that specified in the log file.

\section{6 'Analysis' Module}

The 3D coordinates of gaze points are recorded in the log file using a different node ID (see section 3) for every participant. We present here a part of the analysis process which consists of plotting the gaze points for every subject for the whole length of the session.

In order to add the gaze points to the rendered scene a separate scene graph is created with a root node having as many branches as subjects. The root node is initially set to be invisible to the scene traversal. Every 'subject' branch is successively updated with a sphere placed in the location of every gaze point. Different colours are used for different subjects. This calculation is performed when the log file is first loaded.

When the tape is played for the first time the gaze point scene graph is added to the main scene graph. Pressing a key on the keyboard makes the gaze point root node visible/invisible to the traversal and switches the gaze points on or off. Figure 5 shows a scene rendered with gaze points on. The scene represents a typical setup during three-way multiparty collaboration. It is possible to distinguish gaze points on the avatars' faces and torsos and on the circular table in the middle. Other features will be added to improve the analytical capabilities, including fixation and hotspots maps.

\section{Conclusions and Future Work}

We presented a cross-platform, open-source and lightweight desktop tool that allows analyzing and playing back events of multiparty collaborative sessions taking place in CAVE-like environments and visualizing gaze information. The playback structure is event-replay driven and it optimizes scene graph traversal by implementing a selective update on the most time-consuming commands.

The application distinguishes itself from other playback tools because of its analysis features in $3 \mathrm{D}$, its ability to allow $3^{\text {rd }}$ party view and navigation of the scene during replay, and its portability to other CAVE environments. Future developments will focus on increasing the capabilities of the analysis module, adding audio playback synchronized with the 3D simulation, and porting the player application from desktop to a CAVE environment.
To understand how gaze can affect communication and collaboration in virtual environments the analysis module will be enhanced with features useful to conversation analysis such as information related to participation, and involvement. These aspects can be quantified, for example, by calculating the number of seconds participants are looking at each other or at the background during the session. In this context, more gaze analysis features are being developed to create fixation maps and to highlight areas of interest. Currently audio is only recorded on tape, without using the world-server clock. Future development will concentrate on synchronizing audio with the scene, during replay.

Another possible development concerns porting the current player application from desktop to our CAVElike environment so that users can immerse themselves in the session as invisible observers. This can be implemented with relatively low effort given that our EyeCVE system updating and rendering is also based on the OpenSG library.

Finally, successful analysis of $3 \mathrm{D}$ gaze has implications that go beyond evaluation within our EyeCVE system. In this respect, the tool described here provides a software platform that can be used by other disciplines and transferred to other applications.

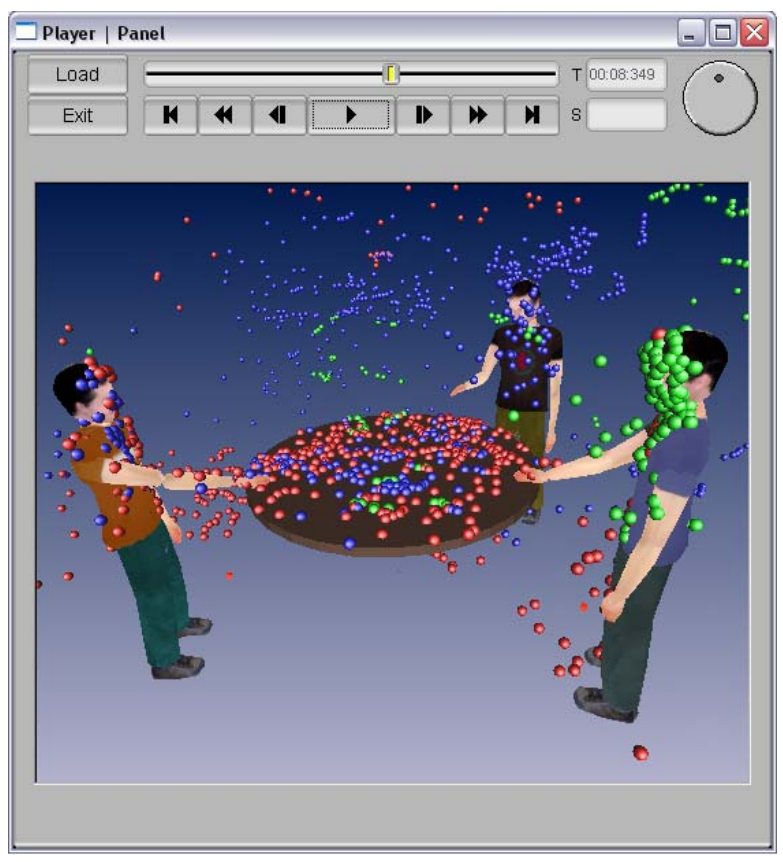

Figure 5. Scene rendered with gaze points on. Gaze points of each avatar are represented using the same shade. Note that the avatars are only shown at one instance of time, whereas the eye-gaze is temporally extended. 


\section{Acknowledgements}

The Eye Catching project (EP/E008380/1) and the EPSRC.

\section{References}

[1] A. Boukerche, D. Duarte, R. Araujo, L. Andrade, and A. Zarrad, "A novel solution for the development of collaborative virtual environment simulations in large scale," presented at Ninth IEEE International Symposium on Distributed Simulation and Real-Time Applications, 2005, Montreal, Canada, 2005.

[2] M. Matijasevic, D. Gracanin, K. P. Valavanis, and I. Lovrek, "A framework for multiuser distributed virtual environments," IEEE Transactions on Systems, Man and Cybernetics - Part B: Cybernetics, vol. 32, 2002, pp. 416-429.

[3] R. Wolff, D. Roberts, A. Murgia, N. Murray, J. Rae, W. Steptoe, A. Steed, and P. Sharkey, "Communicating eye gaze across a distance without rooting participants to the spot," presented at 12-th IEEE International Symposium on Distributed Simulation and Real Time Applications, Vancouver, Canada, 2008.

[4] W. Steptoe, R. Wolff, A. Murgia, E. Guimaraes, J. Rae, P. Sharkey, D. Roberts, and A. Steed, "Eyetracking for avatar eye-gaze and interactional analysis in immersive collaborative virtual environments," presented at Computer Supported Cooperative Work (CSCW08), San Diego, USA, 2008.

[5] H. Sacks, Lectures on Conversation, Blackwell, Oxford, 1995.

[6] V. Hilt, M. Mauve, J. Vogel, and W. Effelsberg, "Recording and playing back interactive media streams," IEEE Transactions on Multimedia, vol. 7, 2005, pp. 960-971.

[7] P. Chiu, A. Kapuskar, S. Reitmeier, and L. Wilcox, "Room with a rear view. Meeting capture in a multimedia conference room," IEEE Multimedia, vol. 7, 2000, pp. 48-54.

[8] Y. Kim, J.-H. Choi, J. Lee, M. K. Kim, N. K. Kim, J. S. Yeom, and Y. O. Kim, "Collaborative surgical simulation over the Internet," IEEE Internet Computing, vol. 5, 2001, pp. 65-73.

[9] C. Greenhalgh, M. Flintham, J. Purbrick, and S. Benford, "Applications of temporal links: recording and replaying virtual environments," presented at IEEE Virtual Reality, 2002, Orlando, FL, 2002.

[10] E. Frécon, "DIVE: communication architecture and programming model," IEEE Communications Magazine, vol. 42, 2004, pp. 34-40.

[11] A. Steed, V. Vinayagamoorthy, and A. Brogni, "Using breaks in presence to identify usability issues," presented at 11th International Conference on Human Computer Interaction, Las Vegas, USA, 2005.

[12] M. Hosseini and N. D. Georganas, "MPEG-4 based recording and replay of collaborative virtual reality sessions," presented at IEEE Virtual Reality, 2002, Orlando, FL 2002.

[13] A. Boukerche, A. R. Lopes, and R. B. de Araujo, "A capture and access mechanism for accurate recording and playing of 3D virtual environment simulations," presented at Tenth IEEE International Symposium on Distributed Simulation and Real-Time Applications, 2006, Torremolinos, Malaga, Spain, 2006.

[14] L. Lambrinos, P. Kirstein, and V. Hardman, "The multicast multimedia conference recorder," presented at 7th International Conference on Computer Communications and Networks, 1998, Lafayette, LA, USA, 1998.

[15] S. Minneman, S. Harrison, B. Janssen, G. Kurtenbach, T. Moran, I. Smith, and B. van Melle, "A confederation of tools for capturing and accessing collaborative activity," presented at Third ACM international conference on Multimedia, San Francisco, California, 1995.

[16] N. Murray, D. Roberts, A. Steed, P. Sharkey, P. Dickerson, and J. Rae, "An assessment of eye-gaze potential within immersive virtual environments," ACM Transactions on Multimedia Computing, Communications, and Applications, vol. 3, 2007, pp. $1-17$.

[17] L. E. Sibert, J. N. Templeman, and R. J. K. Jacob, "Evaluation and analysis of eye gaze interaction," Naval Research Lab Washington DC, 2001.

[18] M. Tory, M. S. Atkins, A. E. Kirkpatrick, M. Nicolaou, and G.-Z. Yang, "Eyegaze analysis of displays with combined 2D and 3D views," presented at IEEE Visualization 2005, Minneapolis, MN, USA, 2005.

[19] A. T. Duchowski, E. Medlin, A. Gramopadhye, B. Melloy, and S. Nair, "Binocular eye tracking in VR for visual inspection training," presented at VRST'01, Banff, Alberta, Canada, 2001.

[20] S. Suzuki, Y. Watanabe, H. Igarashi, and K. Hidaka, "Human skill elucidation based on gaze analysis for dynamic manipulation," presented at IEEE International Conference on Systems, Man and Cybernetics, Montréal, Canada, 2007.

[21] A. Raij and B. Lok, "IPSVIz: An after-action review tool for human-virtual human experiences," presented at IEEE Virtual Reality 2008, Reno, USA, 2008.

[22] E. Gu and N. I. Badler, "Visual attention and eye gaze during multiparty conversations with distractions," Lecture Notes in Computer Science, vol. 4133, 2006, pp. 193-204.

[23] J. M. Findlay and I. D. Gilchrist, Active Vision: The Psychology Of Looking And Seeing, Oxford University Press, Oxford, 2003.

[24] R. Vertegaal, I. Weevers, C. Sohn, and C. Cheung, "GAZE-2: conveying eye contact in group video conferencing using eye-controlled camera direction," presented at CHI 2003, Ft. Lauderdale, Florida, USA, 2003. 